\title{
La espacialización de la pobreza en Bogotá, D.C. 1993-2005
}

\section{Spatial distribution of poverty in Bogotá, D. C. 1993- 2005}

\section{Adelia Hasleidy Sánchez Mariño*}

\section{Resumen}

Son diversos los estudios realizados sobre la pobreza en Bogotá, D.C., enfocados desde las perspectivas política, económica y sociológica, histórica, cultural y artística; algunos de éstos aluden a elementos espaciales de la Ciudad, que han servido como referentes en aspectos específicos del trabajo, objeto de esta tesis, y a los cuales se agradece su valioso aporte. Sin embargo, son pocos los que han asumido de manera estructural el análisis de la pobreza en la Ciudad, desde el enfoque espacial de la Geografía y tal es el propósito de este trabajo. El objetivo de la investigación pretende, por una parte, mostrar el comportamiento de la pobreza en Bogotá, D.C. y propone una metodología de medición más amplia, con nuevas variables, además de las utilizadas actualmente y que permiten ubicar, de manera más precisa, nuevos elementos al conocimiento de la población pobre de Bogotá, D.C.

Palabras clave: Bogotá, D. C., Índice de Necesidades Básica, Índice Sen; Ingreso, pobreza, pobreza extrema. 


\section{Abstract}

There are several studies about poverty in Bogota; nevertheless, most of them are written from the point of view of politics, economy, sociology, history, culture and art. Some of the studies refer to spatial elements of the city, which have been useful in specific aspects of this research. There are few works that have assumed the analysis of poverty in the city from the spatial point of view bestowed by Geography. This is the purpose of this work.

This paper aims to that show the behavior of poverty in Bogota and propose a wider methodology of its measure, by using new variables, other than the traditional way of measuring poverty; in order to locate more precisely the phenomena of poverty and provide new elements to comprehend it in Bogota.

Key words: Bogota, D. C.; Income; Poverty; Capacity, Basic Needs Index; Sen Index. 


\section{Introducción}

Bogotá, D.C., al igual que muchas de las grandes capitales del mundo, se ha convertido en un espacio habitado por población proveniente de diversas regiones del país, población desplazada por la violencia $\mathrm{O}$ migrante movida por imaginarios de progreso y bienestar, además de los que nacen en ella y la han habitado por generaciones. Sin embargo, estos asentamientos se han dado de manera individual, por lo que se ha generado un crecimiento urbanístico acelerado y desmedido, y todos los esfuerzos de la administración por planificar su desarrollo se han quedado cortos ante las crecientes necesidades de la población, por lo que se han producido fenómenos de desigualdad y pobreza, que se han ido acumulando ante las dificultades para brindar asistencia y cobertura de servicios, y ante la carencia de un sistema de análisis y medición efectiva que impide valorar, cuantificar y ubicar con precisión la población de la ciudad.

Este estudio sobre la dimensión espacial de la pobreza en Bogotá, D.C. asume, en primer lugar, aspectos históricos universales del concepto de ciudad, desde sus orígenes hasta el que se tiene actualmente y cómo Bogotá, D.C. responde a ese concepto. En este propósito, para ayudar a comprender la evolución de la ciudad, es necesario vincularla a la forma de producción, que le da una dinámica de funcionamiento económico y cohesión social, que determina, en consecuencia, el ingreso monetario de los individuos y sus familias, y la incidencia de estas condiciones de vida en el espacio que esta población ocupa.

Esta vinculación espacial permite abordar la pobreza no sólo desde la carencia de ingreso económico, sino también desde la negación de derechos y oportunidades de la población.

La estructura tradicional de análisis de la pobreza, de los estudios realizados en Bogotá, D.C. hasta ahora, resulta insuficiente, a juzgar por los resultados obtenidos y en razón a que se ha tenido una visión unidimensional de la pobreza, basada sólo en la ausencia de bienes o carencia de recursos.

Esta tesis articula las diferentes metodologías existentes; la ya tradicional, a partir de las Necesidades Básicas Insatisfechas, NBI, con otras complementarias que trascienden el ámbito de los servicios que provee el Estado y que permiten una mejor aproximación a la comprensión y medición de la pobreza, como son el Índice de Calidad de Vida, el Índice de Pobreza Humana y el Índice de Desarrollo Humano, considerados por Amartya Sen, cuya propuesta se basa en la valoración de los bienes en función de las capacidades de las personas.

El trabajo busca dar una dimensión de análisis integral del fenómeno social de la pobreza, en el que la Historia brinda un apoyo importante para conocer la evolución de la Capital de Colombia, los 
cambios dados, que han contribuido a su construcción y desarrollo hasta lo que es hoy; un resultado que debe entenderse como transitorio, en tanto que producto social cambiante, así como las condiciones que configuran los cambios espaciales y temporales.

El estudio comprende también un análisis de las formas de producción existentes en el marco del sistema de economía de capital y cómo éste ha tenido incidencia en la vida de los bogotanos; sus dinámicas y jerarquías que resultan de estos cambios. Esta parte del estudio es expuesta en el capítulo I y brinda una amplia exposición sobre la manera como la pobreza se manifiesta en el espacio de la Ciudad, desde la óptica de un concepto amplio que va más allá de la simple carencia de recursos económicos, hasta la restricción de oportunidades que acompaña la condición de vida de los habitantes pobres de la Ciudad.

El estudio propone, entonces, una redefinición del concepto de pobreza, desde luego con base en el estudio de Bogotá, D.C., pero que puede tener aplicación en cualquier contexto urbano contemporáneo, donde la condición económica de las personas involucra estados de negación de derechos y de oportunidades, y que, además, afecta la dignidad humana, con unas implicaciones ambientales graves para el espacio habitado; no sólo aquél que ocupan los ciudadanos en condiciones de pobreza, sino también el que ocupan aquellos que no lo son.
Por otra parte, las últimas administraciones de la Ciudad, es decir, desde la de Jaime Castro: "Bogotá para vivir todos del mismo lado", han tenido un especial énfasis en los temas sociales, y es importante destacar el positivo interés en los últimos años, por parte de la sociedad civil, en aportar a este esfuerzo de querer mejorar la calidad de vida de la población de la Ciudad, con iniciativas como "Bogotá cómo Vamos", apoyada por el periódico El Tiempo y la Cámara de Comercio de Bogotá, en asocio con la Alcaldía Mayor de la Ciudad, así como el estudio "Dónde están y Quiénes son los pobres en Bogotá", apoyado por la Fundación Corona y la Alcaldía Mayor; estas campañas constituyen esfuerzos que confirman la prevalencia de la pobreza y la dificultad de implementar estrategias efectivas para disminuirla (Fundación Corona, 2004).

En Bogotá, D.C. y en toda Colombia, se ha institucionalizado la estratificación social, inicialmente con el ánimo de determinar el costo de los servicios públicos de manera acorde con la ubicación espacial, condición socioeconómica de los habitantes y valorización de los predios habitados, pero con el tiempo se convirtió en una forma de administrar, de manera permanente, la inequidad existente en la población, lo cual constituye una aberración política y administrativa en cualquier sociedad democrática.

La dificultad a la que se han enfrentado sucesivos gobiernos, para superar la 
pobreza en la Ciudad, consiste en no haber podido identificar, en forma precisa, la población pobre con los métodos que se han aplicado, de acuerdo con la información obtenida en los informes de desarrollo y en los ejercicios estadísticos, que se concentran en las Necesidades Básicas Insatisfechas y el ingreso económico, tal como se puede observar el las bases de datos del DANE del 2003, que informa sobre el Índice de Calidad de Vida y que no tiene en cuenta variables importantes, como cuantificación de aspectos relacionados con escolaridad y longevidad, que ayudarían a brindar una visión y una medición más precisa de la pobreza en Bogotá, D.C.; éstos son argumentos que fundamentan la importancia de esta investigación y que se corroboran con los resultados alcanzados, como se mostrará más adelante.

La metodología de la investigación permite caracterizar y medir la pobreza en Bogotá, D.C. y es abordada en los capítulos II y III, donde se exponen las características de los tres indicadores compuestos mencionados y cómo éstos determinan los conceptos de bienestar y capacidad, entre otros aspectos, y cómo tales indicadores denotan características espaciales diferentes en cada localidad de la Ciudad.

En el capítulo IV se hace un análisis comprensivo de las características del espacio y las diferentes variables que determinan su valoración y la relación de éste con quienes lo habitan, así como la accesibilidad a los servicios públicos domiciliarios y a los servicios de Educación, Salud, Transporte, Empleo, Seguridad Ciudadana, Ocio y Recreación, calidad de los suelos, condiciones ambientales y densidad poblacional.

Este trabajo se fundamenta, además, en los esfuerzos de varias administraciones que han trazado políticas, planes de desarrollo, programas y proyectos que buscan diagnosticar, identificar y reducir la pobreza en Bogotá, D.C., sin lograrlo de manera efectiva, razón por la cual se hace necesario indagar por qué tales esfuerzos no han sido suficientes $\mathrm{y}$, en no pocos casos, infructuosos, a pesar de los logros alcanzados y buenas prácticas realizadas.

Por otra parte, los trabajos de A. Sen, (Sen, 2000) que hacen parte del soporte teórico y de la propuesta metodológica de análisis de la pobreza en Bogotá, D.C., en este estudio, sólo se conocen en América Latina y especialmente en Colombia después del año 1998, cuando su autor obtiene el premio Nobel de Economía por sus estudios y propuestas alternativas que complementan y enriquecen la visión tradicional de los estudios sobre la pobreza. Por lo anterior, este trabajo de tesis, que involucra los trabajos de Sen, adquiere un valor agregado novedoso.

Además, incorporar a la propuesta de estudio el Índice de Desarrollo Humano de Naciones Unidas, que da prioridad a otros elementos cualitativos para el análisis y medición de la pobreza, cuya 
superación es además una de las prioridades de los Objetivos de Desarrollo del Milenio, ODM, compromiso que Colombia ha asumido junto con los demás países miembros de la ONU, se constituyen en elementos fundamentales y de obligatoria inclusión en todo estudio, y en la metodología de análisis, que conduzcan a la planeación del desarrollo, encaminada hacia la disminución de la pobreza. La siguiente tabla sintetiza los índices y sus variables que serán las herramientas fundamentales del análisis de esta investigación.

Tabla 1. Variables por índice

\begin{tabular}{|l|l|l|}
\hline \multicolumn{1}{|c|}{$\begin{array}{c}\text { Índice de Necesidades } \\
\text { Básicas - INBI }\end{array}$} & $\begin{array}{c}\text { Índice de Pobreza H umana } \\
- \text { IPH }\end{array}$ & \multicolumn{1}{|c|}{$\begin{array}{c}\text { Índice de Desarrollo } \\
\text { H umano - IDH }\end{array}$} \\
\hline VI: Vivienda inadecuada & IL: Índice de longevidad & IEV: Esperanza de vida \\
\hline VS: Vivienda sin servicios & IC: Índice de conocimiento & TA: Tasa de alfabetismo \\
\hline HC: Hacinamiento critico & $\begin{array}{l}\text { TNAA: Tasa de no acceso } \\
\text { agua potable }\end{array}$ & TE: Tasa de escolaridad \\
\hline $\begin{array}{l}\text { DE: Dependencia } \\
\text { Económica }\end{array}$ & $\begin{array}{l}\text { TNAS: Tasa de no acceso } \\
\text { servicio de salud }\end{array}$ & $\begin{array}{l}\text { INE: Indice de nivel } \\
\text { educativo }\end{array}$ \\
\hline $\begin{array}{l}\text { INE: Índice de nivel } \\
\text { educativo }\end{array}$ & $\begin{array}{l}\text { TD: Tasa de desnutrición } \\
\text { ICV: Condiciones de vida }\end{array}$ & II: Índice de ingreso \\
\hline $\begin{array}{l}\text { INE: Índice de nivel } \\
\text { educativo }\end{array}$ & $\begin{array}{l}\text { TD: Tasa de desnutrición } \\
\text { ICV: Condiciones de vida }\end{array}$ & II: Índice de ingreso \\
\hline
\end{tabular}

Fuente: La Autora, con base en PNUD, 2004.

\section{Metodología}

Es importante mencionar que esta investigación se ha restringido a las localidades urbanas de Bogotá, D.C., sin incluir la localidad de Sumapáz, en primer lugar porque la mayor concentración poblacional de la Ciudad se encuentra en las zonas urbanas y Sumapáz es una localidad eminentemente rural y en razón a la ausencia de información estadística adecuada sobre la pobreza en este lugar, tanto en el ámbito nacional, como en las diferentes dependencias de la Alcaldía
Mayor de Bogotá.

Esto no significa que la pobreza esté ausente en esa localidad; por el contrario, su población está constituida mayoritariamente por comunidades que se ubican en los estratos socioeconómicos más bajos y con condiciones especiales, como el desempleo. A su vez, actividades económicas como el sobrepastoreo y la tala de una de las especies vegetales más importantes de su ecosistema de páramo, el Frailejón, son suficientes elementos de juicio para establecer que allí prevalece la pobreza 
e incluso, teniendo en cuenta su clima, existen horizontes preocupantes de miseria en la población habitante.

Esta situación constituye una limitación para hacer un ejercicio en condiciones comparativas adecuadas, como se ha hecho en las localidades urbanas y es, a la vez, un llamado de atención a las autoridades competentes en el ámbito nacional, regional y local, para que se asuma la responsabilidad de elaborar y procesar la información requerida para poder incluir a la localidad de Sumapáz en un estudio científico y sistemático, como se ha podido hacer con la parte urbana de la Ciudad.

Esto, sin embargo, no significa que no exista literatura sobre la pobreza en Sumapáz; existe, pero es insuficiente para aplicar las variables de medición necesarias, razón por la que se ha excluido esta localidad del presente análisis metodológico.

Esta investigación incluye una bibliografía amplia que constituye antecedente importante en diversos aspectos que tienen incidencia en el estudio y comprensión de la pobreza en Bogotá, D.C., su espacialización y cartografía analítica.

En cuanto los componentes que conforman los tres pilares metodológicos de este estudio, además del Índice de Necesidades Básicas Insatisfechas, INBI, están los que determinan la calidad de vida de una persona, propuestos por Sen, (Sen, 2000) y que se resumen en las capacidades, oportunidades y derechos, como elementos fundamentales para la medición, y el Índice de Pobreza Humana, IPH del PNUD, que incluye: la Longevidad, como supervivencia; la Vulnerabilidad, ante la muerte relativamente temprana; el Conocimiento, ante la exclusión de la lectura y la comunicación, y el nivel de vida, relacionado con el aprovisionamiento económico, en términos de acceso a la Salud, Servicios Públicos y nutrición adecuada (PNUD, 2000).

El IPH es desarrollado a partir del Índice de Desarrollo Humano, IDH, y está compuesto de tres indicadores: la Longevidad, como esperanza de vida al nacer: el Nivel Educacional, conformado por la tasa de alfabetización de adultos y la tasa bruta de matriculación combinada: primaria, secundaria y terciaria, y el nivel de vida, medido por el PIB real Per cápita.

Dentro de la bibliografía consultada, las investigaciones de A. Sen constituyen un aporte muy significativo a la propuesta metodológica de integrar varios enfoques de análisis para entender y medir con mayor precisión la pobreza. Sen considera conceptos como las capacidades, oportunidades y derechos: "las capacidades determinan la calidad de vida de una persona; una capacidad es una habilidad o potencial para hacer o ser algo, para lograr un cierto funcionamiento, tan elemental como estar bien nutrido, tener buena salud o tan complejos como alcanzar autodignidad o integrarse socialmente" (Sen, 2000). 
Este enfoque permite un análisis cualitativo en el concepto de pobreza, que ha sido valorado y retomado por el Programa de Desarrollo de las Naciones Unidas, PNUD, en la siguiente definición: "la degeneración de las oportunidades y las operaciones más fundamentales del desarrollo humano, vivir una vida larga, sana y creativa y disfrutar de un nivel decente de vida, libertad, dignidad, respeto por si mismo y los demás" (PNUD, 2004).

Adicionalmente, este enfoque, junto con los mencionados IPH e IDH y el NBI, constituyen la propuesta de análisis integral para identificar la población en condiciones de pobreza en Bogotá, D.C.

Desde el punto de vista del territorio se reconoce que existe una unidad, pero que las condiciones al interior de su extensión difieren en términos de la propiedad y las cualidades espaciales, que generan fragmentación y segregación del espacio, las cuales, sumadas a la existencia de grupos humanos con altas diferencias de ingreso económico, producen una división espacial como resultado de tal situación.

Para hacer el análisis espacial de la pobreza de manera integral, además del estudio de los índices mencionados, se debe considerar el estudio estadístico poblacional de la ciudad, para cuantificar la situación de sus habitantes y establecer las condiciones materiales y la calidad de vida, con el fin de incluirlos ó excluirlos de la situación de pobreza en la ciudad.
Es importante señalar que la información estadística aislada no es suficiente para comprender el problema; es necesario involucrar elementos históricos, teóricos y éticos, para poder comprender, de manera integral, el comportamiento y las manifestaciones de la pobreza en Bogotá, D.C.

La investigación tiene en cuenta dos conceptos que se aplican en sendos métodos de identificación de la población pobre, propuestos por Amartya Sen, en su libro "Desarrollo y Libertad", en el que explica un concepto más amplio de pobreza, que considera las posibilidades y capacidades como factor determinante de dicha condición. De otra parte, Sen define un método directo para medir la pobreza, por medio del INBI u otro indirecto, basado en el ingreso monetario.

Mediante el primero, se determinan como pobres las personas cuyo consumo de bienes deja sin satisfacer alguna necesidad básica (esté método se establece a través de la medición de variables como la vivienda inadecuada, inasistencia a la escuela y alta dependencia económica). Este método tiene un carácter materialista, basado en la canasta de necesidades

básicas de la pobreza.

Por otra parte, el método indirect, consiste en calcular el ingreso mínimo necesario para satisfacer las necesidades básicas, los gastos básicos de alimentación y servicios mínimos; valor que equivale a lo que se conoce como la "línea de pobreza." 


\section{Resultados}

Entre los resultados más importantes de este estudio, se destaca el de lograr una medición, con mayor precisión, del fenómeno de la pobreza, por medio de la utilización complementaria de los índices compuestos, que facilitan un análisis integral y multidimensional de la población pobre de Bogotá, D.C., mediante la integración de los tres índices de medición y sus diferentes variables, sintetizadas en la tabla 1.

Uno de los logros más importante de este estudio es la mayor precisión alcanzada en la medición del fenómeno social de la pobreza en Bogotá, D.C., por medio de la utilización complementaria del Índice de Necesidades Básicas Insatisfechas, utilizado por las instituciones gubernamentales y que se enfoca en los servicios que éstas proveen a los ciudadanos, con el Índice compuesto de Sen y los Índices de Desarrollo Humano e Índice de Pobreza Humana, que se basan en las capacidades, oportunidades, derechos y funcionamientos de los habitantes, para trascender el enfoque unidimensional y materialista y permitir un análisis multidimensional, desde luego, más humano de la pobreza.

Un aporte novedoso de esta investigación ha sido incluir el método de Sen en el análisis y la explicación de los mapas que se presentan a continuación y que muestran gráficamente cómo, según el Índice de Necesidades Básicas Insatisfechas, se presentan resultados altamente favorables para la administración de la ciudad, en la medición de la pobreza (figura 1.) de acuerdo con la medición de 1993, comparada con lo que muestra el mapa de pobreza en 2005. Sin embargo, los Índices de Pobreza Humana y de Desarrollo Humano, contenidos en los figuras 2 y 3 , muestran que, al contrario del INBI, se ha producido un incremento considerable de la pobreza en la ciudad, gracias a la inclusión de otras variables de análisis, que han permitido un estudio comparativo en el período, entre los censos de 1993 y 2005 , más preciso.

Esta visión geográfica permite una mejor comprensión y explicación del fenómeno socioeconómico de la pobreza, a partir de la conceptualización de las diversas relaciones existentes entre la pobreza misma y las múltiples dinámicas que responden a este problema espacialsocial.

Finalmente, este trabajo constituye una herramienta útil para ilustrar a la administración pública sobre la espacialización de la pobreza, y a todos los organismos interesados en reducir este fenómeno, para su erradicación, y para ser tenido en cuenta como una herramienta de planificación y desarrollo, con una dimensión social más comprensiva y humana. 


\section{color}

Figura 1. Comparación por necesidades básicas insatisfechas 1993-2005 (NBI), Izquierda NBI 1993, Derecha NBI 2005

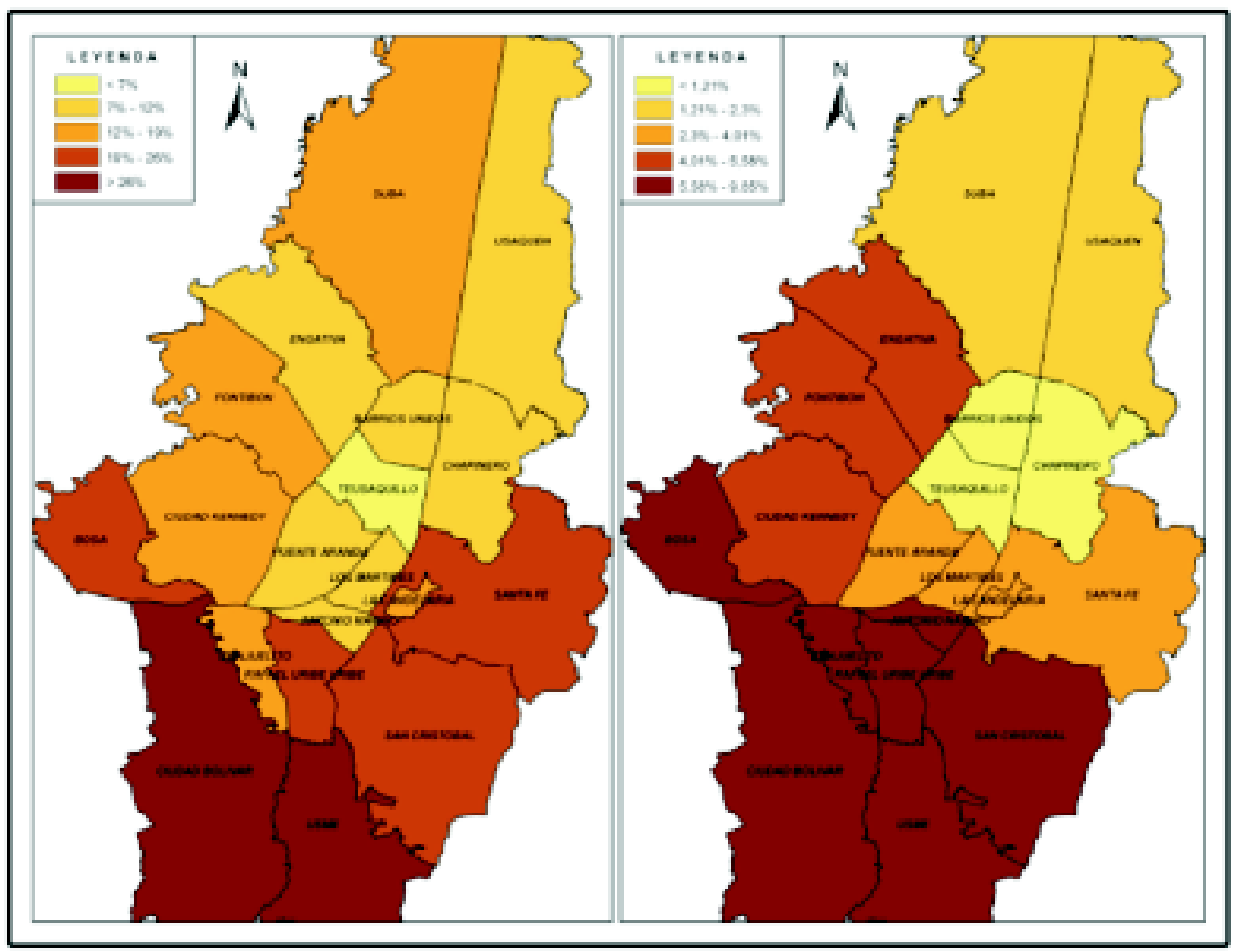

Fuente: la autora, basada en: Censo 1993, Secretaria de Hacienda de Bogotá, D.C., 2005 y DACD, 2005. 


\section{color}

Figura 2. Comparación por Índice de pobreza humana 1993-2005 (IPH), Izquierda IPH 1993, Derecha IPH 2005

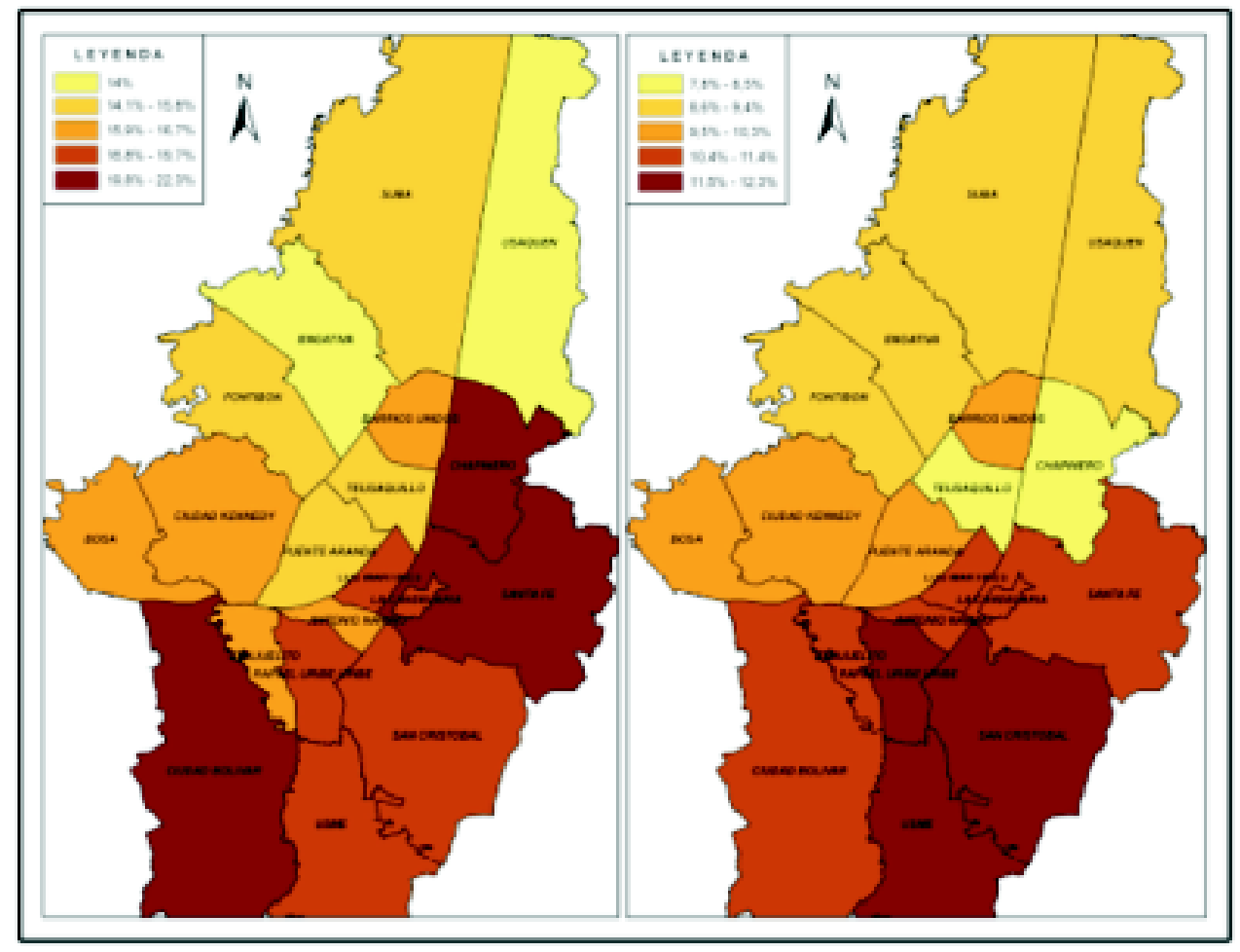

Fuente: la autora, basada en: DAPD 1993, Secretaría de Hacienda de Bogotá, D.C., 2005 y DACD, 2005. 


\section{color}

Figura 3. Comparación por Índice de desarrollo humano 1993-2005 (IDH), Izquierda IDH 1993, Derecha IDH 2005

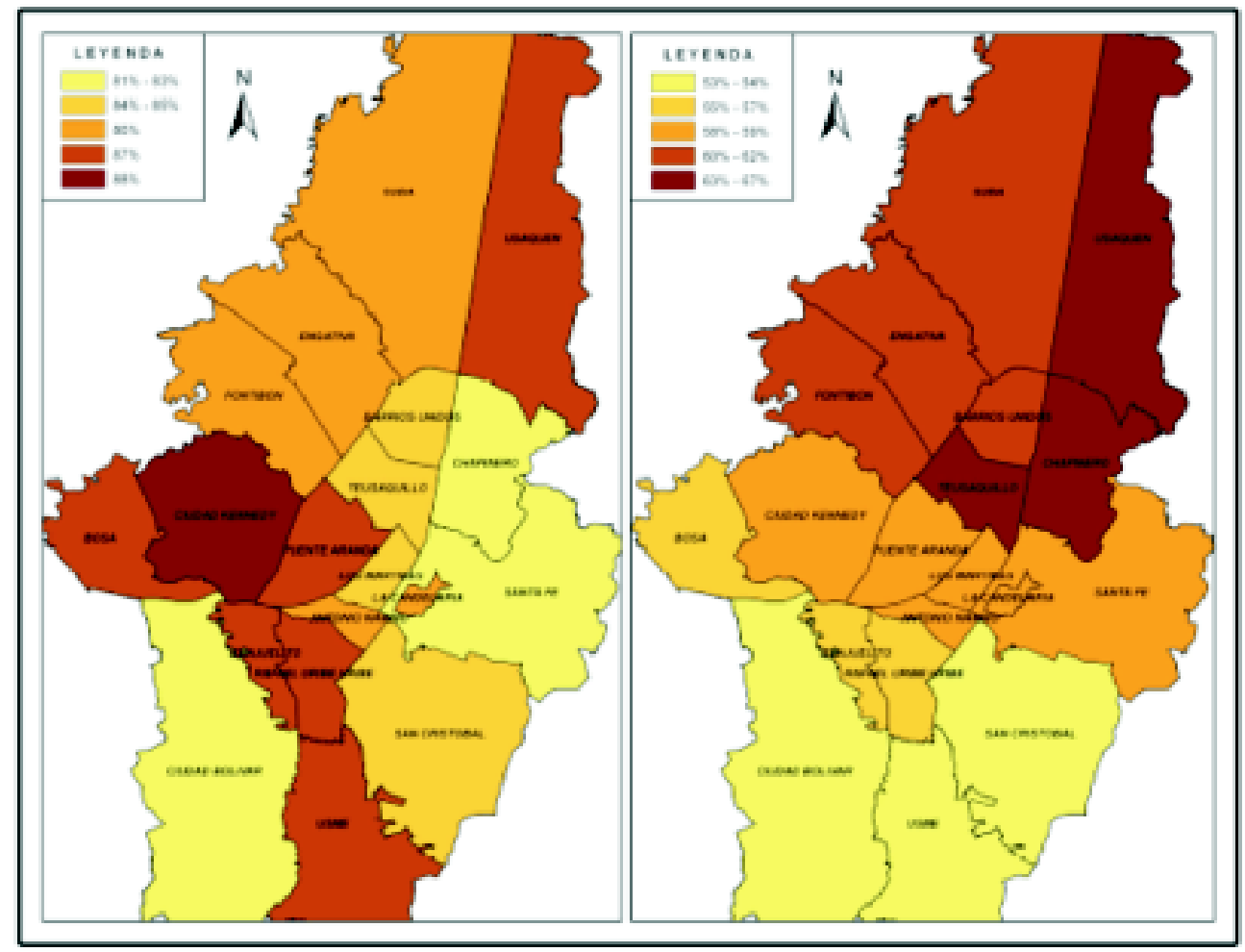

Fuente: la autora, basada en DAPD 1993, Secretaría de Hacienda de Bogotá, D.C., 2005 y DACD, 2005. 


\section{Literatura citada}

DANE. 2003. Índice de calidad de Vida, Bogotá.

Fundación Corona. 2004. El Tiempo. Bogotá cómo Vamos. Alcaldía Mayor de Bogotá.

Fundación Corona. 2004. ¿Dónde están y quienes son los pobres en Bogotá? Ed. El Tiempo, Bogotá.

Harvey, D. 1979. Urbanismo y Desigualdad. Madrid: Siglo XXI. Madrid.

Harvey, D. 2007. Espacios del Capital. Madrid: Akal.

PNUD. 2000. Informe de Desarrollo Humano 2000. Departamento Nacional de Planeación Bogotá.

PNUD. 2004. Informe de Desarrollo Humano 2004. Departamento Nacional de Planeación. Bogotá.

Sen, A. 2000. Desarrollo y Libertad. Ed. Planeta. Bogotá.

Fecha de recepción: 9 de diciembre de 2008

Fecha de aprobación: 29 de diciembre de 2008 